\title{
On the Characterisation of Borderline Cases
}

\section{CRISPIN WRIGHT}

Contents:

$\S 1$ Vagueness-Related Partial Belief

$\$ 2$ Verdict Exclusion

$\$ 3$ Stabilising Liberalism

$\S 4$ Quandary and the Characterisation problem

$\S 5$ The Logic of Vagueness

It is a great pleasure to have the opportunity to contribute to this volume dedicated to the critical celebration of Stephen Schiffer's very considerable philosophical achievements. My focus will be on his recent work on vagueness. ${ }^{1}$

The broad direction of Schiffer's researches in this area has been to give priority to what we may call the characterisation problem: the problem of saying what the vagueness of expressions of natural language consists in or, more specifically - since Schiffer takes it as a given that the vagueness he is targeting consist in a propensity of vague expressions to give rise to borderline cases - the problem of saying what being a borderline case of the concept expressed by a vague expression consists in. This has not been a main preoccupation of most of the work in the field since the vagueness "boom" started in the mid 1970s. There has been a tendency to jump straight into devising semantic theories for vague languages, usually aimed at twin desiderata of saving classical logic and dissolving the various paradoxes of vagueness, with a principal focus on the standard sorites, and occasional glances at the Forced March, and others. ${ }^{2}$ Of course, such work has inevitably implicated commitment to broad conceptions of vagueness, and of borderline cases, of various kinds. The classical epistemicist approach, for example, conceives of borderline cases as instances whose correct classification in terms of the relevant concept is, for reasons it attempts to explain, unknowable. Semantic indeterminist approaches, by contrast, tend (often implicitly) to conceive of borderline cases as items to which the concept in question neither applies nor fails to apply and as coming about because our practice with the concept leaves it, in effect, merely partially defined and so 'gappy'. A variation on this, still semantic indeterminist, regards vagueness as consisting in a phenomenon akin to divided reference, whereby a predicate, for example, may be associated with a range of extensionally distinct best candidates to be the property it refers to; borderline cases are then items which exemplify some but not all of these properties. Finally some have attempted to see vagueness as constituted in rebus - in the world, rather than in meaning or in our ignorance: being a borderline case, so viewed, is a matter of being situated within a penumbra, as it were, like the position of a point between the light and the dark in the image cast by an intense but blurred shadow. ${ }^{3}$ Generally speaking, however, proponents of

\footnotetext{
${ }^{1}$ See the works of Schiffer's cited in the References section. Schiffer's focus, of course, is on soritical vagueness - the kind of vagueness that is characteristic of expressions that are prone to give rise to a sorites paradox. Other linguistic phenomena that are sometimes described as involving vagueness include generality (lack of specificity), partial definition, family resemblances and criterial conflicts. I am concerned only with soritical vagueness in what follows.

${ }^{2}$ For example, the so-called Problem of the Many (for a definitive overview, see Weatherson [2003]).

${ }^{3}$ As far as I am aware, no one has attempted a fully general view of vagueness along these lines.
} 
these various kinds of view have not devoted the same degree of attention to elucidating and defending their (implicit) commitments concerning the nature of vagueness and borderline cases as they have devoted to the development of formal semantical theories, and to criticising opposing views and attempting to address the paradoxes. Yet one would naturally suppose that the characterisation problem should be a locus of developed discussion rather than one of presupposition. For until we have a properly argued account of what vagueness is, how can one possibly expect to know what kind of semantic theory for vague expressions might be best motivated, let alone how the most appropriate kind of semantic theory might assist with the disarming of the sorites paradox and other problems?

I find much to agree with in both the general approach and the details of Schiffer's work on the problems of vagueness. We concur, first and foremost, in prioritising the question of the nature of vagueness and the characterisation of borderline cases - though I confess to being a little less confident than Schiffer that it is possible, or necessary, to accomplish this in an exceptionless, biconditional formulation. We are also agreed in rejecting standard semantic indeterminist, epistemicist and in rebus views, proposing instead that the characterisation of vagueness will best proceed, broadly, in terms of aspects of the distinctive attitudinal psychology involved in the exercise of judgement involving vague concepts. And we are at one in repudiating "third possibility" conceptions of borderline cases: conceptions according to which borderline case propositions - propositions ascribing a vague concept, or its contrary, to items in the borderline area of the concept in question - enjoy a status inconsistent with that of simple truth, or simple falsity. Examples of third possibility status include lack of truth value, the possession of a third truth value, the possession of any of a range of intermediate truth values, and the (dialetheist) idea that they possess both (polar) truth values.

Significant differences, though, remain. Some of the more major concern the epistemic status of borderline cases - in particular Schiffer subscribes to the widespread view of Verdict Exclusion (VE), viz. that (it is known that) no polar verdict about a borderline case proposition is knowledgeable. We also disagree about the kind of attitudinal psychological story to try to tell about borderline cases: Schiffer holds that it should give a central place to his notion of vaguenessrelated partial belief(VPB), although he finds some merit in my notion of quandary; ${ }^{4}$ whereas I would prefer to centralise the notion of quandary, although I think there is insight contained in Schiffer's notion of VPB. Third, although neither of us is prepared straightforwardly to endorse the use of classical logic in reasoning with vague concepts, Schiffer proposes no alternative, holding merely that it is indeterminate whether certain principles of classical logic - including, strikingly, modus ponens - hold good for reasoning among vague judgements, whereas I have argued that the strongest logic justified for such reasoning would involve qualification of the law of excluded middle for atomic statements and of double negation elimination for compound ones (and so approximate an intuitionistic logic).

The considerations to follow will focus on these points of disagreement. They are offered in a spirit of collaboration, in the hope of furthering progress towards the best possible version of the broad genre of account to which Stephen and I are both drawn. ${ }^{5}$

\section{$\S 1$ Vagueness-Related Partial Belief}

It is plausible enough that in the passage along the elements of a typical sorites series for a predicate $\mathrm{F}$, we pass from cases where we are completely confident in the correctness of the

\footnotetext{
${ }^{4}$ More accurately, he finds merit in a notion of quandary in whose characterisation his notion of VPB plays a central role, and which is accordingly rather different from mine.

${ }^{5}$ My own views are principally developed (and developing) in Wright [2001], [2003], [2003a] and [2007].
} 
verdict, "F", through a region where our satisfaction with any particular verdict is qualified and much diminished and then on to a region where confidence builds again in the contrary of the original verdict culminating, as the series progresses, in complete satisfaction once more. I think the phenomenology of whatever it is that seemingly decreases and then increases again in this way needs subtle description, and that it may not be most felicitous to regard it as a form of partial belief. But I don't think Schiffer fundamentally disagrees about that. What is striking is that the attitude involved, although allowing of degree, seems to differ from standard partial belief (SPB) or credence in a number of respects. As Schiffer observes, credences greater than 0 but less than 1 are typically based on evidence which is conceived of as falling short of the best possible evidence, and so are often attended by a conception of how the relevant evidence might be improved. They also tend to be associated with beliefs about likelihood: to believe to some quite high degree that it will rain tomorrow will tend to be to believe (absolutely) that it is quite likely that it will rain tomorrow. Neither of these features is replicated by the kind of partial satisfaction, or confidence, one may have in a verdict that applies a vague concept to a case that lies outside its polar regions but is, say, rather closer to one than the other. But the defining and single most striking feature of VPB is its apparent departure from the laws of classical probability. Classically, my confidence in the conjunction of a pair of (independent) propositions in each of which I place merely partial confidence should approximate the product of the degrees of belief thereby reposed in the conjuncts - and so, when both those degrees are quite small, should be very small. But it does not seem that we would be inclined to regard a conjunction whose conjuncts were a pair of independent propositions, each ascribing a vague property to a more or less "central" borderline case of it, as very much less credible than its conjuncts. Indeed there seems no clear sense in which it would be rational to be any less confident about the conjunction that about the conjuncts. Imagine a cube of a metal which gradually changes colour as it changes temperature - though neither as a result of the other - and suppose it comes to sit simultaneously on the borderlines between red and orange and between warm and hot. Imagine we find we have no preference for the verdict "Cube is warm" over "Cube is hot" and vice versa; and similarly with respects to the verdicts, "Cube is orange", and "Cube is red". It does not seem likely that asked whether we would assent to "Cube is orange and Cube is warm" we would feel any more negative about doing so than about assent to the individual conjuncts. ${ }^{6}$ And no reason is apparent why we should.

The nature of this kind of partial confidence, or satisfaction - for in truth I am not sure how best to represent it - could certainly stand further clarification. What exactly is it that varies in degree, and how may the variation be measured? Betting behaviour, a classic recourse for the explanation of the functional role of standard partial belief, is obviously inappropriate here since there is no question of an 'outcome' where a borderline case is concerned - so nothing to bet on. But although it is arguable that Schiffer has (so far) left issues to do with the functional role and explanatory potential of VPB less clear than is desirable, I think it is hard to dismiss the phenomenon and that the prima facie case for its reality is strong. The question is whether it can take the weight that Schiffer wants to place upon it in his account of vagueness.

About that I continue to have misgivings. Schiffer's strategy is to try to characterise borderline cases directly in terms of the notion of VPB. More accurately, it is to characterise them in terms of the notion of VPB* — vagueness-related partial belief formed under epistemically ideal circumstances. I have already noted elsewhere ${ }^{7}$ certain difficulties with the proposal developed in

\footnotetext{
${ }^{6}$ As Elia Zardini has emphasised to me in conversation, however, Schiffer's point is served merely if any lowering of satisfaction in the conjunction is (appreciably) less than would correspond to the 'multiplication'.

${ }^{7}$ Wright [2006]
} 
Schiffer [2003], to which Schiffer has since responded. ${ }^{8}$ I won't pursue the detail of that particular discussion here, but will outline a more general, though related consideration.

Even if we take the notion of VPB, and its characteristically non-classical behaviour, to be adequately clear, and attested for Schiffer's purposes, any claim that it is of the essence of grasping vague concepts to be prone to VPB with respect to appropriate judgements that involve them looks to be too strong on at least two counts. ${ }^{9}$ Consider first the case of Tim. Tim passes, by any reasonable tests, as a master of a wide range of vague concepts expressible in his natural language. But he has persuaded himself, by a variety of more or less philosophically questionable moves, of the correctness of the classical epistemicist conception of vagueness. Accordingly, he conceives of each of the vague expressions in his language as expressing a property (or other appropriate form of semantic value) that actually has a completely sharply bounded extension. The effect is that although his atomic vague judgements are perfectly orthodox, he is very insistent on the use of classical logic in reasoning with vague judgements and very confident about the principle of bivalence as applied to them. Moreover, and crucially, although his satisfaction with/confidence in the vague (atomic) judgements that he makes varies in degree after the broad manner of VPB, he does - as he should - regard the conjunction of hard cases as increasing the risk of error, and so is prone to "multiply down" - to reject conjunctions in cases where he is not prepared to flat out reject either of the (independent) conjuncts. It would be, I think, strained to insist that Tim's superstitions about the semantics of vague expressions are inconsistent with his perfectly well understanding them - with his fully possessing the concepts that they express. Rather he is making a philosophical mistake - the history of the philosophy of language is littered with such mistakes - about the kind of meanings possessed by expressions which he perfectly well understands. But if that is the right description of him, it is not of the essence of a grasp of vague concepts to enter into attitudes of VPB towards certain judgements involving them.

Alternatively, consider Hugh, an individual who is maximally opinionated. ${ }^{10}$ Hugh's opinions know no half measures. If he takes a view about anything, he takes it with complete conviction. Yet the pattern and spread of his judgements involving vague concepts are otherwise normal. Thus, in the borderline region, of some concept, he sometimes has no view, or returns a verdict inconsistent with one he has given before, - in which case he takes the line that what he said before was "completely wrong". He may even display signs of hesitancy in judging borderline cases - but if he finally overcomes it and is moved to judgement, that judgement is once again completely confident. In short, while the extensional profile of Hugh's judgements involving vague concepts is normal, there is none of the phenomenology of partial belief. And again, it would seem an overreaction to the case to view this psychological quirk as calling into question Hugh's grasp of the concepts concerned.

I think these cases show that while Schiffer is correct to have emphasised the role of something akin to partial belief in the normal attitudinal psychology of vague judgement, - and deserves credit for the insight that this kind of partial belief may, perfectly rationally, display the non-classical features he emphasises, - it is wrong to see it as belonging to the nature of the understanding of vague concepts to involve a disposition to partial belief of this kind, or to write it into the possession-conditions for vague concepts that one should be so inclined.

\footnotetext{
${ }^{8}$ Schiffer [2006]

${ }^{9}$ I take Schiffer to be suggesting such a claim in his [2006] though it is another question whether he needs to.

${ }^{10} \mathrm{I}$ am indebted here to Elia Zardini.
} 


\section{$\S 2$ Verdict Exclusion}

"Verdict" is here a term of art. A verdict about a statement involving a vague concept is a judgement that it takes one in particular of the two polar values, true and false. The thesis of Verdict Exclusion (VE) is that for borderline cases of the concept concerned, no such verdict can be an expression of knowledge. Concerning (VE) itself, three possible views are relevant: (i) that it is known to be false - so known that knowledgeable verdicts, of truth or falsity respectively, are possible for, say, predications of a vague concept of its borderline cases; (ii) that it is known to be true - so known that borderline cases are cases where knowledge of the relevant kind of verdict is impossible; and (iii) agnosticism - that neither view about the status of (VE) is mandated.

Schiffer's view is (ii), a contention that he finds intuitive and regards as enforced in any case by the role of VPB in the individuation of borderline cases. ${ }^{11} \mathrm{I}$, by contrast, believe that the correct stance overall is (iii): we should be agnostic about the possibility of knowledgeable verdicts in borderline cases.

The disagreement is crucial. Verdict Exclusion is no less a nodal issue for the proper characterisation of vagueness than Third Possibility, in whose rejection Schiffer and I, as noted, concur. In my view, a repudiation of both Third Possibility and Verdict Exclusion - that is, a rejection of both theses as unjustified - belongs with a more general liberal stance concerning the epistemic nature of borderline cases. In this section, I will outline some motivations for liberalism and defend it against certain objections, including some of Schiffer's. However what I take to be Schiffer's principal reason for endorsing (VE) - and hence for rejecting liberalism - has to do with his conception of VPB as independently excluding potentially knowledgeable belief. ${ }^{12}$ The issues around that impress me as very difficult, and I must reserve discussion of them for another occasion.

Schiffer and I are both impressed by the datum that ordinary speakers do not treat the borderline area of a vague distinction as one where competence mandates silence or suppression of any inclination to offer a verdict, however qualified. If something is on the borderline between red and orange, say, it won't call your competence with those concepts - or your eyes — into question if you are inclined to describe it as red, or for that matter as orange. One is entitled, if one is so moved, to a verdict in the borderline area, so entitled — presumably - to the opinion which that verdict expresses.

A perhaps more forceful expression of this entitlement intuition - since free of any demand on one's preferred understanding of "borderline cases"-is this. Consider any typical soritical series running from clear instances of $\mathrm{F}$ to clear instances of non-F by steps small enough to service a sorites paradox as plausible as any. Then - this is the intuition - there will be no element of this series about which it is mandatory to return neither the verdict " $F$ " nor the verdict "non-F". A polar verdict is always permissible provided it is sincere; there are no cases that mandate a third possibility type of response. If someone prefers to understand "borderline case" in such a way that the knowledge that something is a borderline case ought to inhibit any verdict, then a way of expressing the entitlement intuition is that there are no clear-definite - borderline cases in a typical sorites series. The clear cases on the other hand are those where only one polar verdict is permissible.

Liberalism is the simplest theoretical accommodation of the entitlement intuition. It is the view that it is always permissible to return a verdict about a borderline case simply because it is -

\footnotetext{
${ }^{11}$ See Schiffer [forthcoming], sections 6 and 7

${ }^{12}$ I take his view to be that the sort of qualified acceptance involved in VPB-ing P to some significantly greater degree than not-P is categorically unsuited to serve as the doxastic ingredient in knowledge.
} 
in a sense we need to clarify - open what to think about such cases and open, indeed, whether in thinking one thing in particular, you are knowledgeable. This openness is, at a minimum, what goes with agnosticism about Third Possibility and Verdict Exclusion respectively: if neither thesis is justified, there is no call to stifle aspects of our natural practice and inclinations to judgement that would be inappropriate if they were true. ${ }^{13}$

Let me quickly summarise some considerations which I take to support liberalism and then -in the next section - respond to some objections to it. First, to emphasise the implausibility of the idea of a datum of Third Possibility. If Third Possibility were known to obtain, even if in just one case, we would know that any polar opinion about that case was mistaken. But we do not behave as though we think we know any such thing: someone who returns a (perhaps suitably qualified) polar verdict about a borderline case is never thereby automatically treated as revealing a mistake, or incompetence - rather we feel, to repeat, that they are, ceteris paribus, precisely entitled to their opinion. The manifestations of judgement of borderline cases include hesitation, inability to form an opinion, weakness of opinion, instability of opinion, and conflict among judges whose competence is not in question. But convergence in patterns of hesitation, or non-opinion, still less any sense that 'no opinion' is sometimes the uniquely appropriate response, is at best contingently (and doubtfully) involved. Mastery of a vague concept seems to involve no essential exercise of a concept of any kind of third possibility.

Now to Verdict Exclusion. If (VE) were known to obtain, even if in just one case, we would know that any polar opinion about that case was, whatever else, not the product of a successful feat of cognition. It would be something caused, no doubt, by relevant features of the case but not a fitting cognitive response to them. And if (VE) we known to obtain generally in the borderline area, then our propensity to verdicts - albeit weak and unstable verdicts - about cases lying within it would seem to amount to no more than a kind of cognitive incontinence. It seems that to know such a thing about opinions one is inclined to form should have the effect of undermining them. So knowledge of Verdict Exclusion is also in tension with the entitlement intuition. Our sense is that no matter what case in a sorites series we consider, it is consistent with full perceptual and conceptual competence if someone takes a (perhaps suitably qualified but) polar view of it. There are no cases, even towards the 'middle', where it is eo ipso incompetent to have a (suitably qualified) polar view. However a presumption of knowledgeability - or at least warrant - is a condition of rational opinion: for a rational judge, judging that $\mathrm{P}$ is judging that $\mathrm{P}$ is what one ought to think. But one who thinks that (VE) is known says in effect that we know that there is no mandate for opinion in some cases in a sorites series - there is, in borderline cases, nothing that ought to be thought. In such cases, thinking that $\mathrm{P}$ is what one ought to think, is therefore mistaken. (VE) thus has the effect that opinions which, according to the entitlement intuition, are consistent with competence, are ungrounded and hence not competent. If (VE) is part of the best theory of vagueness, then the best theory is one according to which our actual practice in all but definite cases is irrational/incompetent. It is hard to envisage that a best theory should have that feature.

Finally, there are difficulties for (VE) involved in its interaction with principles of Evidential Constraint (EC). The best formulation of the latter for any particular type of judgement is doubtless going to be controversial. But (EC) is undeniably intuitive for secondary qualities generally and for a wide class of predicates of casual observation - "heap", "bald", etc., — that

\footnotetext{
${ }^{13}$ In refusing to affirm that no verdict about a borderline cases can be knowledgeable, the liberal does not, of course, intend to exempt such verdicts from other normal controls on knowledge ascription - the point is only: we have no justification for thinking that knowledgeability is excluded just by a verdict's concerning a borderline case.
} 
foster sorites paradoxes. We are not, intuitively, up for the idea of baldness that cannot be recognised as such in principle, or heaps whose heaphood would elude the detection of even the most fortunately situated judge. Yet if Verdict Exclusion were known to obtain, even if in just one case, we would know that (EC) - in the form of the two conditionals:

If $\mathrm{P}$, then it is feasible to know $\mathrm{P}$

If not-P, then it is feasible to know not-P

- would have to fail in that case (on pain of the obvious contradiction.)

This, it seems to me, is simply too strong a result to swallow. Maybe (EC) is controversial, ${ }^{14}$ and perhaps it is implausible for certain vague expressions. ${ }^{15}$ But it is already a problem if it is not implausible for all - since our knowledge of (VE) is being supposed to be characteristic of borderline cases without exception: if (VE) and (EC) are (known to be) inconsistent, the plausibility of taking (VE) to be known for an arbitrary vague predicate, say, is hostage to that of taking it that we actually know that (EC) fails for it. The proponent of (VE) owes an explanation of what he takes the content of this knowledge to be. And if it is, as naturally construed, that colours, baldness, heaps and so on can all be undetectable, even under unimprovable conditions of observation, then I do not think we do know anything of the sort, for a very wide class of vague concepts. ${ }^{16}$

\section{$\S 3$ Stabilising liberalism}

On the other side, there are a number of more or less intuitive objections to liberalism, some amounting to direct arguments for (VE). While the matter requires a much fuller treatment than I have space for here, I'll try to address at least some of the anti-liberal considerations known to me and point up some of the issues on which further clarity is needed.

\footnotetext{
${ }^{14}$ It is, of course, in tension everywhere with Williamson's thesis that knowledge is subject to margins of error in a sense that requires that knowing that $\mathrm{P}$ holds of circumstances $\mathrm{C}$ entails that any case within some fixed margin of difference of $\mathrm{C}$ is also a case where $\mathrm{P}$ holds. I'll come back to this below.

${ }^{15}$ Schiffer [forthcoming] suggests "brave".

${ }^{16}$ Schiffer [forthcoming] suggests that the motivation for EC confuses the above conditionals with the (in the relevant, plausible cases) acceptable weakenings:
}

If Definitely $\mathrm{P}$, then it is feasible to know $\mathrm{P}$

If Definitely not-P, then it is feasible to know not-P

This is no help, however without a developed account of the difference in the truth conditions of the original and weakened formulations, and a story about how exactly the original versions are supposed to fail. Recall that, as noted in the main text, even the supposition that we do not know that the original conditionals fail is inconsistent with the claim that (VE) is known.

Here is a related point, due to Zardini. Consider the claim - with which the epistemicist is comfortable, though not, I would imagine, Schiffer - that some nth case in the series is such that either it is F (say, looks red) and we cannot feasibly know that or it is not-F and that we cannot feasibly know that. Formally,

$(\exists$ n $)\{($ Fn \& $\sim$ it is feasible to know $(\mathrm{Fn})) \mathrm{V}(\sim \mathrm{Fn} \& \sim$ it is feasible to know $(\sim \mathrm{Fn}))\}$

That is something which, if we do not believe that there is any such n, we may very well want to deny. Intuitionistically and classically, the denial is equivalent to:

(*) $\quad(\forall \mathrm{n})\{\sim(\mathrm{Fn} \& \sim$ it is feasible to know $(\mathrm{Fn})) \& \sim(\sim \mathrm{Fn} \& \sim$ it is feasible to know $(\sim \mathrm{Fn}))\}$

And $(*)$ is already enough to yield aporia when conjoined with (VE). (VE) is inconsistent with the denial that there are any elements in a sorites series for looks red which look red but cannot be known to do so, or which don't look red and cannot be known to do that. If (pace the epistemicist) one can rationally doubt there are any such elements, one cannot coherently endorse (VE). 
Schiffer himself ${ }^{17}$ offers two intuitive thoughts on behalf of (VE). The first, very simply, is that

"it's as much of a platitude to say that

If someone knows that $\mathrm{S}$, then it's determinately true that $\mathrm{S}$

as it is to say that

If someone knows that $\mathrm{S}$, then S."18

Assuming the standard kind of characterisation of S's being borderline, viz. that it is neither determinately true that $\mathrm{S}$ nor determinately true that not-S, it follows that no-one ever knows a borderline S. But I reply that Schiffer's platitude is clearly that only if "determinately" carries the sense of a kind of particle of emphasis, like "actually' in one common kind of use, or "indeed". When it is so understood, to characterise a borderline case for $\mathrm{S}$ as a situation where it is neither determinately true that $\mathrm{S}$ nor determinately true that not-S is, in effect, to endorse Third Possibility - which Schiffer does not.

Schiffer's second intuitive thought invites us to reflect

"on one's epistemic position when confronted with what one knows to be a borderline proposition. Suppose that you are holding a ball in your hands in circumstances that are as good as you can conceive of them getting for judging the ball's color. You are certain you know what color the ball is, whether or not you have a word for that color in your vocabulary; you know that you have mastery of the concept of red; and you know with certainty that the color you know the ball to have is not one you can now justifiably say either is or is not red. Furthermore, you cannot even conceive of how, given all you know, you could come to have warrant for judging either that the ball is red, or that it isn't red. You rightly take yourself to be in the best possible position to verify whether or not the ball is red, and you can't imagine what you could conceivably find out that would give you knowledge that the ball was, or wasn't, red. Given all that, I would think that you would be entirely justified in thinking that it's impossible, given the obtaining facts, for you, or anyone else, to know whether or not the ball is red. Examples involving any other sorites-prone concept can be used to make the same point."19

I think the portion of this up to but excluding the last two sentences nicely characterises some of the phenomenology of quandary. ${ }^{20}$ It is indeed in such a situation hard to conceive of any improvement in one's epistemic position which one could foresee would sway the balance. But to find oneself unmoved to an opinion by evidence of which one has no clear conception of a possible improvement justifies the claim that there is no knowing in the case in question only if one knows, first, that one's lack of a clear concept of a possible improvement is an indicator that there is no possible improvement - which raises interesting issues which I will not here go into-and, more crucially, second, that one's present evidence is not enough for knowledge: that had one been moved to a verdict on the basis of the very same experience and collateral beliefs, one's present quandary discloses that being so moved would have been inappropriate, rather than the other way round. But you don't know that. Had you in the same circumstances been moved - marginally - to the opinion that the ball is red, it would go with that opinion to think that coming to no view in the same circumstances would-marginally-underplay the evidence. Do you know now that that would be an inappropriate reaction?

\footnotetext{
${ }^{17}$ Schiffer [forthcoming], p. ??

${ }^{18}$ Schiffer [forthcoming], p. ??

${ }^{19}$ Schiffer [forthcoming], p. ??

${ }^{20}$ Although the bit about knowing "with certainty that the color you know the ball to have is not one you can now justifiably say either is or is not red" is too strong.
} 
Here next is an argument ${ }^{21}$ that purports to commit liberalism to an actual contradiction. Suppose that Aye and Nay come to different (but suitably qualified) polar verdicts about a borderline case of F. Liberalism - specifically, agnosticism about (VE) - seems committed to saying that it is not known that Aye is not knowledgeable, and it is not known that Nay is not knowledgeable. But this pair of claims, given only uncontroversial proof-theoretic properties of knowledge, is unstable. For the latter claim seems to imply that

$$
\sim \mathrm{K}(\sim \mathrm{K} \sim(\mathrm{Fk})) \text {, }
$$

and the former that

$$
\sim \mathrm{K}(\sim \mathrm{K}(\mathrm{Fk}))
$$

And from these, given factivity and closure for ' $\mathrm{K}$ ', we get, by elementary moves, that $\sim \mathrm{K}(\mathrm{Fk}) \& \sim \mathrm{K} \sim(\mathrm{Fk})$

So: on the - apparently-liberal supposition that it is not known that either polar verdict is not knowledgeable, we appear to have shown that neither polar verdict is knowledgeable! This conclusion will go for all polar disagreements about borderline cases. So in no such disagreement is any knowledgeable opinion involved, just as VE requires, and contrary to liberalism.

I think the right reaction to this objection is that it points up the need for a distinction in the interpretation of the various occurrences of the operator " $\mathrm{K}$ ". Liberalism indeed cannot be coherently expressed otherwise. But what distinction? The general idea is that we try to be open to the possibility that any particular opinion about a borderline case may be knowledgeable, including both one's own, if one has one, and that, possibly conflicting, of others. But obviously, if Aye's opinion is that $\mathrm{P}$, then she ought to think that anyone who thinks otherwise is wrong, so not knowledgeable. What liberalism requires is that, consistently with thinking that he doesn't know, Aye should somehow nevertheless allow that Nay could be knowledgeable, even thinking what he actually does in the world as it actually is. What is the modality there?

It's the same as that whereby, when you hold any opinion of which you are not entirely sure, you may concede, consistently with retaining that opinion, that you could be wrong. I carefully count the marbles in a bag, and get a largish number. If I am right about the number, then given the way I arrived at my belief - by a careful count - it seems reasonable to say that I know what it is. But perhaps you nag me that even careful counts can involve error when largish numbers are involved, and I am thereby moved to concede that I could be wrong. What is the content of that concession? It had better not be tantamount to the admission that I do not know, after all, my belief may be true and formed by careful execution of a reliable, indeed canonical method. So the concession needs to be weaker. But nor does it seem entirely happy to view me as admitting that I do not know that I know; for if we are understanding knowledge in enough of an externalist way to allow that I may still actually know the number of marbles in the bag, the thought invites itself that I may still (so to say, externally) actually know that I know that I do. A better suggestion is that what I concede is the right to claim to know. I opine that $\mathrm{P}$ but I do not claim to know that $\mathrm{P}$, though nor do I admit that I do not know it.

So, let's try that distinction in the context of Aye and Nay: Aye's opinion that P commits him to holding that Nay does not know not-P; but Nay's knowing not-P is nevertheless consistent with everything that Aye regards herself as in position to claim to know-since she does not regard herself as in position to claim to know P. Nay's knowing not-P is a possibility for Aye in that somewhat qualified sense of epistemic possibility. Again, this is not the same as saying that it is consistent with everything Aye in fact knows - not if one thing she may in fact know is $\mathrm{P}$.

\footnotetext{
${ }^{21}$ From Rosenkranz [2005]
} 
The crucial thing is thus that, when moved to a verdict in the borderline area, one is in no position to claim to know (although still in position to consider that one may know.) This requires, to stress, that the things which one is in position to claim to know are a potentially narrower class than those which in fact one does know. Indeed it requires, I readily acknowledge, a more general account of the notion of one's "being in position to claim" and an explanation of its potential to carry a narrower extension than one's actual knowledge. I do believe in the good standing of and need for such a notion, but I cannot argue for that here. ${ }^{22}$

To review the original argument in the light of this distinction. Let "RP" express that one is in position to claim to know $\mathrm{P}$. Then the ingredients in the liberal supposition become

$$
\begin{aligned}
& \sim \mathrm{R}(\sim \mathrm{K} \sim \mathrm{P}), \text { and } \\
& \sim \mathrm{R}(\sim \mathrm{KP})
\end{aligned}
$$

Given that $\mathrm{R}$ is closed, (and $\mathrm{K}$ factive) we still get

$\sim \mathrm{RP}$ and $\sim \mathrm{R} \sim \mathrm{P}$

But that is now no problem-just the (agnostic) result that no one is in position to claim knowledge of a verdict in a borderline case.

To summarise, both the following liberal-seeming claims -

1. No-one is in position to know that any polar verdict about a borderline case is, just in virtue of its subject matter and specific polarity, not knowledgeable.

2. No-one should commit themselves to thinking that any particular polar verdict about a borderline case is, just in virtue of its subject matter and specific polarity, not knowledgeable -

are incoherent and are no part of the commitments of liberalism. The first commits one who endorses it to the premises of the Aye-Nay reductio. The second is inconsistent with the entitlement intuition, since anyone who takes a polar view of a borderline case naturally commits themselves to thinking that the opposing view is false, and therefore not knowledgeable. What, I am suggesting, is a commitment of liberalism is this

3. No-one is in position to claim to know that any polar verdict about a borderline case is, just in virtue of its subject matter and specific polarity, not knowledgeable.

We are entitled, when so moved, to have polar opinions about borderline cases. When we do, we are committed to thinking that contrary such opinions are not true, and so not knowledgeable. But we are in no position to claim to know that a given such opinion is not true, or not knowledgeable. And we are also in no position to claim to know that a given such opinion is true, or is knowledgeable.

There is one final powerful-looking extant argument in favour of (VE), and hence antithetical to liberalism. It is Timothy Williamson's argument that inexact knowledge requires a margin for error, with the latter notion understood in such a way as to entail that cases within a fixed margin of difference from a case known to be $\mathrm{F}$ are likewise $\mathrm{F}$. This enforces the negation of the claim that all the EC-conditionals of the form,

If $\mathrm{Fk}$, then it is feasible to know that $\mathrm{Fk}$,

\footnotetext{
${ }^{22}$ It is argued for in Wright [forthcoming].
} 
for $\mathrm{k}$ an element in a given sorites series, are true. ${ }^{23}$ It dos not, except classically, enforce the idea that some in particular are false; it does not, except classically, even preclude thinking that there is none that is false; not does it force us to conceive of the falsity of such a conditional as consisting in the F-ness of the relevant $\mathrm{k}$ but unfeasibility of knowledge that Fk. There is therefore a project, for those, like the present author, for whom intuitionistic distinctions will be respected in the logic of choice for vague statements, of exploring whether or the extent to which Williamson's argument might somehow be stopped short of full out contradiction with liberalism. But my own expectation is that something will have to give here. Williamson's proposal is driven by two thoughts: (i) that knowledge requires reliability of the relevant method of belief formation, and (ii) that in creatures of limited powers of discrimination, reliable detection of a characteristic will be compromised if changes too slight for them to discriminate can make the difference between its applying and its failing to apply. There is evident merit in both thoughts. Where there may be room for manoeuvre is over whether, as best understood, they properly combine to enforce the general truth of Williamson's putative corollary, that cases within a fixed margin of difference from a case known to be $\mathrm{F}$ are likewise $\mathrm{F}$. But the matter needs a careful separate treatment - something I cannot embark on here. ${ }^{24}$

Specific arguments apart, it is clear that there is a strong intuitive pull, felt by many philosophers of very different theoretical predispositions about vagueness, to accept (VE). What is the root of it? One motivation, undoubtedly, is the intuitive predilection for Third Possibility conceptions of borderline cases. Another is the questionable idea that knowledge requires subjective certainty, coupled with the thought that the borderline region is one of often tentative, uncertain opinion. A third would regard the characteristic instability of opinions in the borderline region as disqualifying them as knowledge - but that wouldn't justify the modal component in (VE): those drawn to it are unlikely to feel any better about a verdict about a borderline case which just happened to be stable. My guess is that the single most powerful pre-theoretic motive for endorsement of (VE) is the idea that the opposed contention, that knowledge in borderline cases is possible, owes a concrete conception of a number of matters that present as moot, to say the least - indeed as imponderable. What kind of fact could it be that a case on the borderline of "red" is actually red? - won't one need recourse to something approaching the epistemicist's idea of inscrutable semantic mechanisms linking the predicate to a property of which we may have no adequate conception to make sense of such a fact? And if one goes in that direction, what would make an opinion that happened to coincide with the fact knowledgeable?

In short, it looks as though a worked out denial of (VE) will involve all the problems that discourage most from endorsing epistemicism, together with the additional baggage of explaining how the kind of sublimated facts in which epistemicism believes are in principle open to knowledge. But if this is the primary, most basic motive for endorsing (VE), it should come with a sense of liberation to realise that a justifiable recoil from this cluster of issues and problems should actually motivate no such thing. We are not, in denying that (VE) is justified, affirming its negation. We incur none of the distinctive obligations of that affirmation. The liberal view is that we do not know (VE); but nor do we claim to know that knowledge is possible in the borderline area. We have, accordingly, no obligation to provide a further account of the facts of which it would be knowledge or of how knowledge of them might be reached.

\footnotetext{
${ }^{23} \mathrm{We}$ assume that each successive element in the sorites lies within the relevant margin of difference of its predecessor.

${ }^{24}$ Useful discussion bearing on the issue may be found in Mott [1998], Williamson [2000b], and Egré [2006]
} 


\section{$\$ 4$ Quandary and the Characterisation problem}

I turn to the question of whether the notion of quandary might be usefully deployed to address the characterisation problem. An early decision needs to be taken about the form in which to try to address the problem, since there are several interrelated questions here. They include:

(1) In what does the vagueness of a vague expression consist?

(2) What is a borderline case object/item?

(3) What is a borderline proposition?

One natural proposal is to try to answer (3) first, after which the answers to questions (1) and (2) can respectively consist in elaboration of these two basic thoughts:

(1) That the vagueness of E consists in the fact that its presence in token utterances results, in certain circumstances, in their expression of borderline propositions;

(2) That a borderline case item is the subject of a borderline proposition.

The following remarks are offered under the aegis of this natural proposal.

In earlier work, I characterised the notion of a quandary as follows: ${ }^{25}$

Proposition $\mathrm{P}$ presents a thinker $\mathrm{T}$ with a Quandary in circumstances (of evaluation) $\mathrm{C}^{26}$ if and only if, in $\mathrm{C}$,

(i) $\mathrm{T}$ does not know whether or not $\mathrm{P}$

(ii) $\mathrm{T}$ does not know any way of knowing whether or not $\mathrm{P}$

(iii) $\mathrm{T}$ does not know that there is any way of knowing whether or not $\mathrm{P}$

(iv) $\mathrm{T}$ does not know that it is (metaphysically) possible to know whether or not $\mathrm{P}$

I also suggested that it would be wrong to add

(v) T knows that it is not (metaphysically) possible to know whether or not $\mathrm{P}$

for reasons included in and elaborated upon by the discussion of the preceding sections. It is a consequence of the proposal sans clause (v) that the less cognitively adept a thinker, the easier it will be to confront her with quandary - since she only has not to know various things. Indeed she doesn't even have to be able to wonder whether or not P. Clearly there is therefore no prospect of capturing the notion of a borderline proposition by reference to the propensities of actual thinkers to be put in quandary by it. We will need to consider thinkers of some degree of conceptual and cognitive sophistication.

A first shot at a characterisation in terms of quandary of a borderline proposition might run as follows:

$\mathrm{P}$ is borderline in circumstances $\mathrm{C}$ iff a conceptually and perceptually fully competent thinker $\mathrm{T}$ could be put in quandary by $\mathrm{P}$ in $\mathrm{C}$

The intuitive thought is that, while quandary is never a mandated response to a borderline situation, it is always a possible one quite consistently with full and proper cognitive functioning and grasp of all relevant concepts. In a clear case, by contrast, to fall into quandary is not

\footnotetext{
${ }^{25}$ Wright [2001]

${ }^{26}$ The relativity to circumstances of evaluation is for the obvious reason: the very same proposition can be a quandary in one set of circumstances and clearly true in another. But the relativity might be compounded for vagueness-unrelated reasons if one's conception of proposition allows propositional truth to be relative to time, or standards, etc.
} 
consistent with full and proper cognitive functioning in appropriate respects together with grasp of all relevant concepts, since the clear cases mandate a verdict. Notice that this strategy of characterisation grants that quandary is not a characteristic mental state associated with the appraisal of a borderline proposition - not if "characteristic" means widespread and typical. It is the possibility of quandary, consistently with full competence in all relevant respects, that is crucial, not the actual prevalence of it.

The first shot characterisation, however, is still manifestly insufficient. The problem is that there are non vagueness-related quandaries. A thinker of arbitrary conceptual and perceptualindeed, mathematical - competence may very well meet all four clauses with respect to Goldbach's Conjecture, for example: a proposition in which there is no relevant form of vagueness, and which accordingly has no claim to be a borderline proposition. And moral dilemmas hold out the prospect of another region of quandary: finding myself in a situation in which all possible courses of action conflict with one or another of my values, I may consider myself apprised of all relevant non-moral facts and quite reasonably be perfectly agnostic about the prospects of adjudicating between the competing values concerned. In that case P, i.e., some proposition of the form: "A is the best thing to do in the circumstances", may very well present me with a quandary. True, there are almost certain to be vague concepts involved in P; but it seems likely to be a misdiagnosis of the source of the quandary posed by a moral dilemma to locate it there.

Goldbach's conjecture and the wider class of propositions that it represents-propositions, whether or not mathematical, for which we have no assurance of decidability - are, I think, easy to exclude by a well-motivated modification of the first shot proposal. What is striking about these cases is that quandary is mandated - a fully competent thinker ought to regard Goldbach as presenting a quandary. That is in crucial contrast to the situation of borderline cases, at least as construed by liberalism. So it looks as though the first shot should be followed by a second shot beginning like this:

$P$ is borderline in circumstances $C$ iff

(i) a conceptually and perceptually fully competent thinker T could, consistently with those competences, be put in quandary by $\mathrm{P}$ in $\mathrm{C}$; and

(ii) Such a $\mathrm{T}$ is not required to be put in quandary by $\mathrm{P}$ in $\mathrm{C}$; and....

- though only so beginning, since something now needs to be added to address the quandary of moral dilemma. There are other possible quandaries too: if one is persuaded of the possibility of faultless disagreement in matters of taste, for example, there looks to be potential for quandary in the situation of a bystander considering which of the protagonists in such a disagreement may be right.

There is now a tactical issue. We could proceed by trying to track through all possible varieties of quandary, hoping to find a clause for each to distinguish it from the vagueness-related cases. But it is not clear what degree of insight might be expected along that path. Better, if we can, to say something which captures the nature of vagueness-related quandary in one cast, as it were, thereby systematically contrasting it with all the other kinds. How might that be accomplished?

Here is a tempting thought: perhaps this is the place to re-invoke VPB. It is not implausible that either of the conflicting claims in a moral dilemma might - by one for whom it is not a dilemma-be endorsed as strongly as you like without any necessary implication of some form of incompetence. Likewise for the ingredient claims in a putatively faultless disagreement on a matter of taste. But can any thinker who is fully competent in relevant respects endorse a verdict about a vague judgement strongly - as strongly as you like - which, for another relevantly fully competent thinker, presents a quandary? In a borderline case, where quandary is consistent with competence, 
must not that fact surface in at least some, perhaps quite significant degree of qualification in the confidence of one who, as according to liberalism she is entitled, endorses a verdict? If so, we can add to the second shot along the following lines:

(iii) $\mathrm{T}$ is required to repose at most a relatively low degree of confidence (VPB) in P (or its negation) in C, and hope thereby to have at least the overall shape of a successful characterisation, (even if further refinement will certainly be needed.)

However there is, if an earlier point is correct, a problem for this - the same problem caused by our friend Hugh and the possibility of "maximal opinionation" for the general idea that VBP is a (non-contingently) characteristic mental state of vague judgement. If maximal opinionation is consistent with mastery of vague concepts, VPB is at most contingently so characteristic - and the requirement proposed in clause (iii) as formulated is not a requirement. I see no way round this objection at present.

What we should like to propose would be a clause that captured the idea that in borderline cases, a thinker who is put into quandary will be so because of the nature of the prevailing circumstances of evaluation and the vagueness of the judgement concerned: the quandary is an upshot of the vagueness. But of course to say that would be to surrender the project of accounting for the nature of vagueness in terms of the propensity to induce quandary in fully competent thinkers - rather we will have slipped into thinking of vagueness as an underlying cause of the attitude of quandary, whose nature will therefore have to be explained independently. ${ }^{27}$

Surely, though, the basic thought has to be right that it goes with the nature of the kind of vagueness we are concerned with that quandary may be induced without demanding explanation by defective cognitive or conceptual competence. And when quandary is so induced, it is presumably induced by something. The project of attempting a broadly attitudinal-psychological response to the characterisation problem means that we cannot rest content with an account of the cause that involves an unreconstructed appeal to the vagueness of the proposition concerned. But it may quite properly appeal to a certain kind of characteristic property of the relevant circumstances of evaluation - if we can find one. And surely we can. The very form of the sorites paradox itself provides the means. All the vague concepts with which we are concerned are, after all, soritesprone - that defines the kind of vagueness with which we are concerned. And every sorites paradox involves gradual change in the values of some parameter, $\Pi$, with the following characteristic: that there is a finite strict ordering of such values whose early instances provide for clear cases of $\mathrm{F}$ and whose later ones for clear cases of its contrary. The kind of quandary distinctive of vagueness is one induced by this process of gradual change - or more accurately, since quandary is not restricted to soritical contexts, one induced, in the circumstances in question, by the value for the relevant parameter taken by the object of the judgment that presents a quandary. Call such a parameter a parameter of supervenience for F. For bald, a parameter of supervenience will be number and distribution of hairs on the scalp; for heap of sand, a parameter of supervenience will be number and arrangement of grains of sand; for red, a parameter of supervenience will be colour. I take it these ideas are sufficiently clear for the present purpose. Perhaps, then, the following clauses point in a potentially profitable direction:

$\mathrm{P}$ is borderline in circumstances $\mathrm{C}$ iff $\mathrm{P}$ configures some concept $\mathrm{F}$ for which $\Pi$ is a parameter of supervenience such that

\footnotetext{
${ }^{27}$ Schiffer confronts an exactly analogous problem when he speaks of VPB* as "F-concept driven" in borderline cases - for development of the challenge, see my [2006]. Schiffer responds in Schiffer [2006].
} 
(i) A conceptually and perceptually fully competent thinker T could be put in quandary for $\mathrm{P}$ in $\mathrm{C}$ by the value taken by $\Pi$ in $C$; and

(ii) A conceptually and perceptually fully competent thinker $\mathrm{T}$ is not required to be put in quandary for $\mathrm{P}$ in C.

However it is important to keep in mind the general pressures from which this proposal springs. In general there is no hope, I think, of a successful characterisation of vagueness in terms of the attitudinal states of those who make vague judgements unless we include some form of causal constraint on the provenance of these states. Any successful such account will therefore be bipartite: it will proffer some putatively distinctive attitudinal feature(s) of vague judgement and it will tie significant instantiations of it/them to causes that are somehow an essential feature of the broader landscape of vague judgement. What I have suggested in this section is merely a rather simple-minded illustration of this model: the selected distinctive feature is the association of vagueness with judgemental paralysis in certain circumstances - glossed as quandary - and the relevant feature of the broader landscape is the value taken by the parameter, gradually shifting in a suitable sorites paradox, on which instantiation and non-instantiation of the relevant vague concept supervenes.

It remains to observe that if VPB is a well-conceived phenomenon at all, - which I do not doubt, - then there has of course to be scope for other versions of this general form of bipartite proposal which seek to centralise it, rather than quandary as characterised. Such versions will presumably be more congenial to Schiffer and may well have advantages. I regard the area as very open.

\section{$\S 5$ The Logic of Vagueness}

There is no immediate connection between the proposal that borderline cases should somehow be characterised in terms of a certain distinctive kind of partial belief and a treatment of the sorites paradox. Schiffer effects one by proposing a simple set of characterisations of the degrees of $\mathrm{VPB}$ - degrees of V-credibility - that a rational subject will assign to compounds of vague statements on the basis of the degree of V-credibility that she assigns to their constituents. The matrices he proposes follow the pattern of Lukasiewicz's tables for infinitely many valued logics. Thus the V-credibility of a negation is 1 minus the V-credibility of the negated statement; the Vcredibility of a conjunction is the minimum of the V-credibilities of its conjuncts; and the Vcredibility of a disjunction is the maximum of those of the disjuncts. The universal and existential quantifiers respectively take the greatest lower bound and least upper bound of the V-credibilities possessed by their instances.

Now consider a sorites paradox, with a major premise taken in the form, $\sim(\exists x)\left(F x \& \sim F^{\prime}\right)$. Classically (and intuitionistically) this forms an inconsistent triad with the two minor premises, F0 and $\sim \mathrm{Fk}$, with k selected so as to ensure that the latter is effectively incontrovertible, while F0 is beyond dispute. So it is natural to think that first base for any solution must be to acknowledge that the sorites reasoning reduces the major premise to absurdity and so demonstrates $\sim \sim(\exists \mathrm{x})(\mathrm{Fx}$ $\& \sim \mathrm{Fx}^{\prime}$ ). The question is then how to block the classical entailment of the unwelcome 'unpalatable existential', $(\exists x)\left(F x \& \sim F^{\prime}\right)$, or somehow make out that it is not really unpalatable. The landscape changes however with Schiffer's proposals. This becomes clear if we ask what degree of $\mathrm{V}$-credibility attaches to the unpalatable existential. It will be the maximum of the V-credibilities of the $\mathrm{k}$ instances: conjunctions of the form, $\mathrm{Fx} \& \sim \mathrm{Fx}^{\prime}$, whose $\mathrm{V}$-credibility in turn will be the minimum of those of their conjuncts. In the polar regions this will be very low, since one or the other conjunct will be roundly disbelieved. But the figure will climb as one enters the borderline 
region, culminating, at least in principle, with a V-credibility of, or very close to .5 (though never higher.) So the unpalatable existential, and hence also its negation - the major premise for the paradox - will also have V-credibility very close to .5. The upshot is that, according to the Lukasiewicz-style matrices, both are paradigms of indeterminacy.

Is that a good result? It may seem not - after all, the major premise is very plausible; that is why we had a paradox. So don't we want it to turn out to have quite a high VPB? Well, yesinasmuch as we want to explain the plausibility of the major premise (in particular, its plausibility over its negation) but - you might suppose - also no, since we have to fault it somehow.

Suppose - as I take it Schiffer intends - that the computation we have just sketched is meant to be normative: to deliver a measure of VPB that it is rational to have. Had the computation delivered the result that the major premise did have quite a high VPB, then the account would be saying that we ought to accept it over its negation - in effect, confirming the rational acceptability of the premises of the paradox! So it is good that we don't get that result. But as it is, the result is that rationally there is absolutely nothing to choose between the major premise of the sorites and its negation. That leaves the pull that the premise exerts on us-which is the whole source of the paradox - as unexplained, indeed as irrational. And surely, one may protest, it is not irrationalafter all, the negation is tantamount to assertion of the existence of a sharp cut-off, i.e. a denial of the vagueness of $\mathrm{F}$ in the series in question, which is a datum of the problem.

Why on Schiffer's account does the negative existential form of the major premise for the Sorites attract us? Rationally, if his matrices for VPB are on the right lines, we should find nothing to favour it over its negation. I am not sure how Schiffer sees the options here. One thing he can say is that we are under no pressure to choose - since the law of excluded middle, conceived as a general schema, is foursquare indeterminate too (an exercise for the reader.) That suggests the following diagnostic: the major premise attracts us because acceptance of its negation repels. But the truth is that there is no rational pressure to accept either if the law of excluded middle is indeterminate. However this leaves unexplained why the unpalatable existential is unpalatable. For if it really is tantamount to a denial of the vagueness of $\mathrm{F}$ in the series in question, it is false, not indeterminate. And if it isn't, the repulsiveness of that denial doesn't explain its unpalatability.

How exactly, in any case, does getting in position to regard the major premise as indeterminate dispose of the paradox? It may seem obvious: the paradox was that we seemed driven to a contradiction from acceptable premises - the contradiction, e.g., that a man with exactly 37 cents both is not - of course - and is - by the sorites - rich. Now, since one of the sorites premises turns out to be indeterminate, we are no longer under any pressure to accept one of the components in the contradiction.

What is salient, though, — and of course Schiffer is absolutely aware of and explicit about this - is that that cannot be his whole story. There is a commitment in his account to a kind of over-kill. Not merely have we disposed of the acceptability of the major premise. We have also got into a position where we have to regard it as indeterminate whether the sorites reasoning is valid. For since the minor premise is true, and the conclusion is false, and since - at least for Schifferthe indeterminacy of $\mathrm{P}$ is to be consistent with the truth of $\mathrm{P}$, the situation is one where it is consistent with everything we know that the sorites premises are both true and its conclusion false - so it's consistent with everything we know that it is an invalid argument. A corollary, as Schiffer observes, is that - since we may run the sorites just using hundreds of special conditional premises instead of the usual major premise-we have to regard the validity of modus ponens as an indeterminate issue too! Is this something that can be lived with?

There may be some temptation to reply that Schiffer's account treats the validity of modus ponens, or other classical rules, as indeterminate only when we are involved with indeterminate 
premises and/or conclusions. Elsewhere there is no problem. But I don't think such an attempt to ring-fence the singularity makes sense. Validity - at least, classical validity - turns on compossibilities of truth-value. If certain argument patterns have instances involving indeterminate premises and false conclusions, - or more graphically, true premises and indeterminate conclusions $^{28}$ - then they are not known to be truth-preserving. So why should we trust them anywhere? And in any case, don't we need reliable rules in terms of which to bring out the commitments of a thinker who takes a view-however qualified - on an indeterminate issue?

I have little space to pursue this fundamental matter further. To be sure Schiffer's predicament - if that is what it is - is a function of his choice of the Lukasciewicz-style matrices, and they have independent discomforts in this setting; for example, they mispredict the level of partial belief which normal thinkers will repose in conjunctions of vague but incompatible conjuncts, and they predict variations in the V-credibility of conjunctions of the form, Fk and $\sim \mathrm{Fk}^{\prime}$, $\mathrm{k}$ and $\mathrm{k}^{\prime}$ adjacent in a sorites series, which are surely not empirically confirmed - one would expect a uniformly low valuation, irrespective of the place in the series of $\mathrm{k}^{29}$ But the point I would like to emphasise in closing is that the position for which Schiffer proposes to settle is one in which he has, given other things he accepts, to doubt that certain classically valid inferences are knowledgepreserving. The inference for example, from $\mathrm{F} 0$ and $\sim \mathrm{Fk}$, for suitably 'distant' $\mathrm{k}$, to the relevant unpalatable existential is classically valid and has - one would suppose - known premises. But the conclusion, on Schiffer's calculation, is indeterminate - so cannot be claimed to be known. Moreover if with Schiffer, we accept (VE), we will have to say that it cannot be known. In that case - whatever the situation with truth - classical validity fails to preserve knowledge.

Strikingly, that is, in effect, exactly the intuitionists' complaint about it: that it permits the derivation from warranted premises of conclusions for which there is no warrant- in particular, none elicitable from the warrant for the premises and the derivation. My own treatment of this agenda is precisely fashioned around argument for and consequences of the thesis that classical logic is epistemically non-conservative where vagueness is concerned. Schiffer's views commit him to the same. What he has yet to provide is his own account of the shape a logic should assume to remedy the defect.

The forgoing has concentrated on points of disagreement and difference. It would be entirely inappropriate to end on anything but a note of admiration for the work I have been commenting on. For the philosopher who wishes to better understand the nature of linguistic competence and linguistic representation, vagueness presents challenges of exceptional importance and the greatest intellectual difficulty. Stephen Schiffer has responded to these challenges with a rare mix of breadth of philosophical vision, resourcefulness and dialectical and technical expertise. I look forward to the products of his continuing engagement. ${ }^{30}$

\footnotetext{
${ }^{28}$ Consider the inference from the minor premise and negation of the conclusion of the Sorites to the negation of the major premise.

${ }^{29}$ Elia Zardini has suggested in discussion that a restriction of the Lukasiewicz clauses to independent propositions would be a natural and attractive way of trying to get around these awkwardnesses, (assuming a case can be made that $\sim \mathrm{Fk}^{\prime}$ is relevantly negatively dependent on Fk.)

${ }^{30}$ I am most grateful to Elia Zardini for detailed comments on the penultimate draft, and to him and other members of the Arché Vagueness project for feedback on various of the ideas herein canvassed at various project seminars over the last few years.
} 


\section{REFERENCES}

P. Egré [2006] "Reliability, Margin for Error and Self-Knowledge" 2006, in V. Hendricks and D. Pritchard, eds., New Waves in Epistemology, Ashgate

P. Mott [1998] Margins for Error and the Sorites Paradox", The Philosophical Quarterly 48, 494-504

S. Rosenkranz [2005] 'Knowledge in Borderline Cases', Analysis 65, 49-55

S. Schiffer [1998] 'Two Issues of Vagueness', The Monist 88, 193-214

[2000] 'Vagueness and Partial Belief', Philosophical Issues 10, 220-57

[2000b] 'Replies to Commentators, Philosophical Issues 10, 321-43

[2003] The Things We Mean, Oxford: the Clarendon Press

[2006] 'Replies', Philosophy and Phenomenological Research 72, 233-43

[forthcoming] 'Quandary and Intuitionism: Crispin Wright on Vagueness' in A. Miller, ed., Logic, Language and Mathematics:

Essays for Crispin Wright Volume II, Oxford: Oxford University Press

B. Weatherson [2003] "The Problem of the Many" in the Stanford Encyclopaedia of Philosophy at http://plato.stanford.edu/entries/problem-of-many/

T. Williamson [2000] Knowledge and Its Limits, Oxford: Oxford University press

[2000b] "Margins for Error: A Reply”, The Philosophical Quarterly 50, 76-81

C. Wright [2001] “On Being in a Quandary: Relativism, Vagueness, Logical Revisionism”, Mind 110, 45-98

[2003] "Vagueness: a Fifth Column Approach" in J.C Beall and Michael Glanzberg, eds., Liars \& Heaps: New Essays on Paradox, Oxford: Oxford University Press, 84-105

[2003a]"Rosenkranz on Quandary, Vagueness and Intuitionism", Mind 112, 465-74

[2006] 'Vagueness-related Partial Belief and the Constitution of Borderline

Cases', Philosophy and Phenomenological Research 72, 225-32

[2007] “ 'Wang's Paradox' ” in the Library of Living Philosophers volume for Michael Dummett 
[forthcoming] "Externalism and Scepticism" in a Journal of Philosophy special number on Epistemic Norms, edited by J. Collins and C. Peacocke 